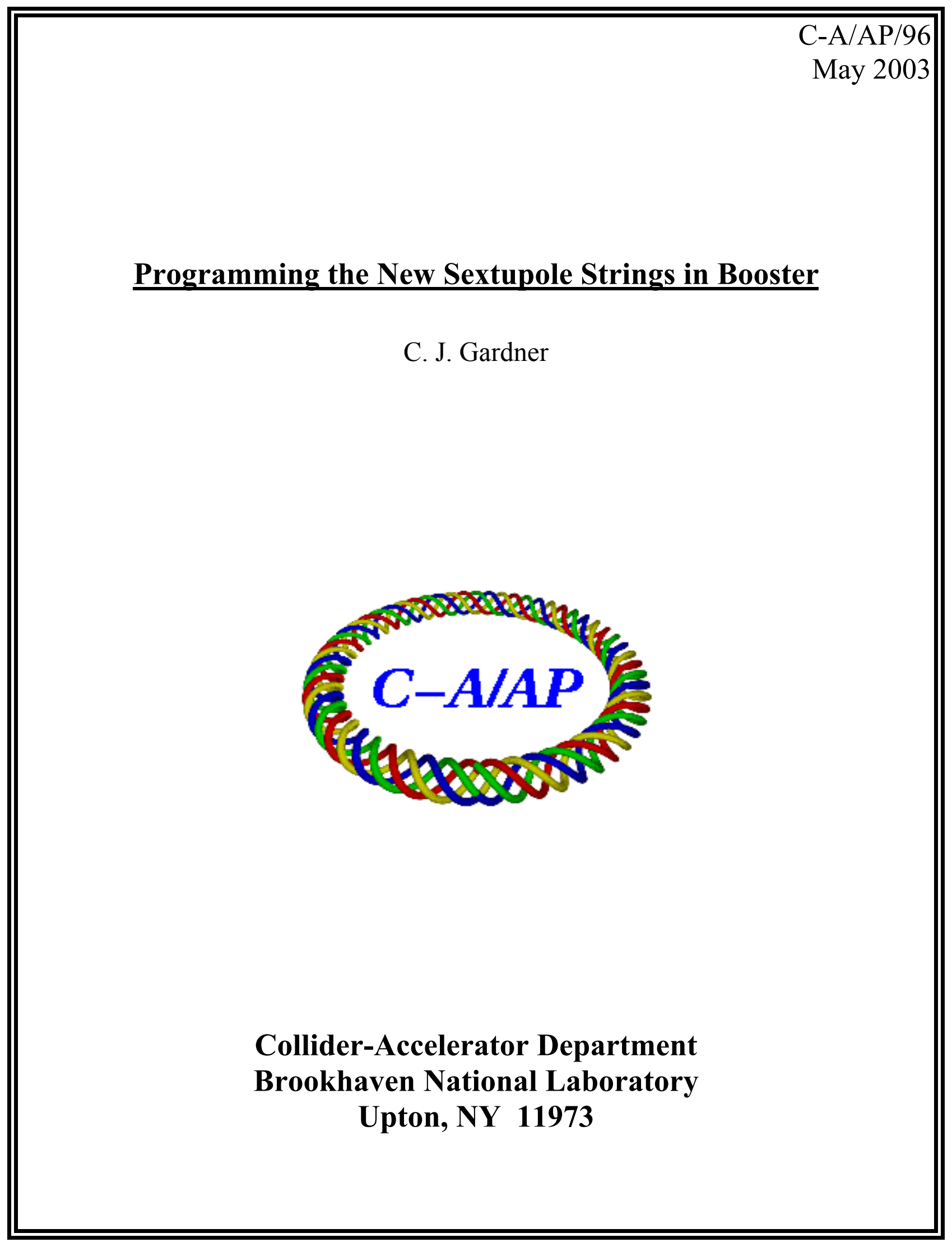




\title{
Programming the New Sextupole Strings in Booster
}

\author{
C.J. Gardner
}

May 5, 2003

The sextupoles in Booster have been reconfigured into a new set of strings to allow for the resonant extraction of beams to the new Booster facility NSRL (National Space Radiation Laboratory). Following is a description of the new strings and their programming for chromaticity adjustment and resonant extraction.

\section{The Sextupole Strings}

Each superperiod of Booster contains eight sextupoles which are labeled SVX1, SHX2, SVX3, SHX4, SVX5, SHX6, SVX7, and SHX8, where SH and SV denote, respectively, sextupoles located near horizontal and vertical beta maximums, and X refers to superperiod A, B, C, D, E, or F. We shall refer to the $\mathrm{SH}$ and SV sextupoles as horizontal and vertical sextupoles respectively. The sextupoles have a main winding consisting of 8 turns, a monitor winding consisting of one turn, and an auxiliary winding consisting of either one or two turns. The main windings are connected together to form four series strings called the Horizontal and Vertical strings and the $\mathrm{C}$ and $\mathrm{F}$ strings. The Horizontal and Vertical strings are used for chromaticity adjustment; the $\mathrm{C}$ and $\mathrm{F}$ strings are used to excite the $3 Q_{H}=13$ resonance for resonant extraction. ( $Q_{H}$ and $Q_{V}$ are the horizontal and vertical tunes, respectively.) Within each string, all sextupoles are excited with the same polarity.

The vertical string contains all 24 of the vertical sextupoles and remains in its original configuration. The horizontal string used to contain all 24 of the horizontal sextupoles, but now four of these, SHC8, SHF8, SHB4 and SHE4, have been taken out to form the $\mathrm{C}$ and $\mathrm{F}$ strings. With these four sextupoles removed, there are four "holes" in the horizontal string, each hole having a partner located three superperiods away. This arrangement 
of horizontal sextupoles is depicted schematically in Figure 1. Here the open red circles show the positions of the four holes; the filled circles show the positions of the remaining 20 horizontal sextupoles.

The horizontal and vertical strings are powered by programmable monopolar power supplies that can deliver a maximum current of $300 \mathrm{~A}$ at a maximum of $90 \mathrm{~V}$. The polarity of the strings is such that current from the horizontal supply increases the horizontal chromaticity, while current from vertical supply increases the vertical chromaticity. According to the definitions of Ref. [1], this means that current from the horizontal supply puts the sextupoles of the horizontal string in "B" polarity, while current from the vertical supply puts the sextupoles of the vertical string in "A" polarity. The wiring diagrams for the horizontal and vertical strings are shown in Figures $\mathbf{2}$ and $\mathbf{3}$. Here the letter "T" denotes the magnet lead that is physically stamped with the letter "T". In accordance with the definitions of Ref. [1], a positive current into the "T" lead puts the magnet in "A" polarity; a negative current into the lead puts the magnet in "B" polarity.

The main windings on sextupoles SHC8, SHF8, SHB4 and SHE4 are connected together to form the $\mathrm{C}$ and $\mathrm{F}$ strings. The $\mathrm{C}$ string contains sextupoles SHC8 and SHE4; the F string contains SHF8 and SHB4. The two strings are depicted by the blue lines in Figure 1. Each string is connected to its own 350 A bipolar programmable power supply as shown in Figure 4. Here, in accordance with the conventions of Ref. [1], a positive reference at the power supply input produces a positive current out of the terminal labeled " 1 ". This puts the sextupoles in the strings in "B" polarity. The $Q_{H}=4+1 / 3$ resonance is excited by powering the two strings with opposite polarity. Note that in the control system, the power supplies for the C and F strings are called "b-sxr1-ps" and "b-sxr2-ps" respectively.

\section{Harmonics Produced by the Holes}

The superperiod symmetry of the machine implies that the holes in the horizontal string will produce only even harmonics in azimuthal angle $\theta$ around the ring. Although this ensures that the $3 Q_{H}=13$ and

$Q_{H}+2 Q_{V}=13$ resonances (which are excited by harmonic 13) will not be excited, the $3 Q_{H}=14$ and $Q_{H}+2 Q_{V}=14$ resonances (which are excited by harmonic 14) can be excited. Note, however, that because the four 
holes are approximately equally spaced in betatron phase and in azimuth $\theta$, harmonics $2,6,10,14,18$, and so on, are suppressed to some extent. To ensure that the $3 Q_{H}=14$ and $Q_{H}+2 Q_{V}=14$ resonances are not excited during injection and acceleration, the currents in the four resonant extraction sextupoles will track the current in the horizontal string until extraction time.

\section{Calculation of Chromaticities, Resonance Parameters and Currents}

Let $I_{H}$ be the current in the string of 20 horizontal sextupoles.

Let $I_{V}$ be the current in the string of 24 vertical sextupoles.

Let $I_{C}$ be the current in the $\mathrm{C}$ string.

Let $I_{F}$ be the current in the $\mathrm{F}$ string.

Then the horizontal and vertical chromaticities are given by

$$
\xi_{H}=\xi_{H}^{0}+\frac{S}{B \rho}\left(K_{H} I_{H}+K_{V} I_{V}+K_{C} I_{C}+K_{F} I_{F}\right)
$$

and

$$
\xi_{V}=\xi_{V}^{0}+\frac{S}{B \rho}\left(L_{H} I_{H}+L_{V} I_{V}+L_{C} I_{C}+L_{F} I_{F}\right)
$$

where $\xi_{H}^{0}$ and $\xi_{V}^{0}$ are the bare chromaticities, $S$ is the integrated strength per unit current of each sextupole, and $B \rho$ is the magnetic rigidity. The constants $K_{H}, K_{V}, K_{C}, K_{F}, L_{H}, L_{V}, L_{C}$, and $L_{F}$ are derived in Appendix I. Note that, as defined by (1) and (2), the bare chromaticities are what one would measure with zero current in the four sextupole strings. The values to be assigned to $\xi_{H}^{0}$ and $\xi_{V}^{0}$ are discussed in Appendix II.

It is convenient to parameterize $I_{C}$ and $I_{F}$ as

$$
I_{C}=I_{H}+I_{0}+I_{R}, \quad I_{F}=I_{H}+I_{0}-I_{R}
$$

where

$$
I_{0}=-I_{H}+\left(I_{C}+I_{F}\right) / 2, \quad I_{R}=\left(I_{C}-I_{F}\right) / 2 .
$$

The currents $I_{R}$ and $I_{0}$ are responsible respectively for the excitation of the $3 Q_{H}=13$ resonance and the excitation of sextupole harmonics $0,4,8$, 12, 16, and so on. (As noted in Section 2, harmonics 2, 6, 10, 14, 18, and 
so on, are suppressed to some extent by superperiod symmetry.) This suggests that we define resonance parameters

$$
R_{0}=\frac{S}{B \rho} K_{0} I_{0}, \quad R=\frac{S}{B \rho} K_{R} I_{R}
$$

where the constants $K_{0}$ and $K_{R}$ are chosen to give $R_{0}$ and $R$ convenient units. Thus, the four physics parameters controlled by the sextupole strings are $\xi_{H}, \xi_{V}, R_{0}$, and $R$.

If the four currents $I_{H}, I_{V}, I_{C}$, and $I_{F}$ are specified, then $\xi_{H}, \xi_{V}, R_{0}$, and $R$ are calculated according to equations (1), (2), (4), and (5). Conversely, if $\xi_{H}, \xi_{V}, R_{0}$, and $R$ are specified, then the four currents can be calculated as follows. Using (3) in (1) and (2), we have

$$
\Delta_{H}=\left(K_{H}+K_{C}+K_{F}\right) I_{H}+K_{V} I_{V}+\Gamma_{H}
$$

and

$$
\Delta_{V}=\left(L_{H}+L_{C}+L_{F}\right) I_{H}+L_{V} I_{V}+\Gamma_{V}
$$

where

$$
\begin{gathered}
\Delta_{H}=\frac{B \rho}{S}\left(\xi_{H}-\xi_{H}^{0}\right), \quad \Delta_{V}=\frac{B \rho}{S}\left(\xi_{V}-\xi_{V}^{0}\right), \\
\Gamma_{H}=\left(K_{C}+K_{F}\right) I_{0}+\left(K_{C}-K_{F}\right) I_{R},
\end{gathered}
$$

and

$$
\Gamma_{V}=\left(L_{C}+L_{F}\right) I_{0}+\left(L_{C}-L_{F}\right) I_{R}
$$

The currents

$$
I_{0}=\left(\frac{B \rho}{S K_{0}}\right) R_{0}, \quad I_{R}=\left(\frac{B \rho}{S K_{R}}\right) R
$$

are obtained from equation (5). Using these in (9) and (10), one obtains $\Gamma_{H}$ and $\Gamma_{V}$ in terms of $R_{0}$ and $R$. Equations (6) and (7) then may be solved to obtain $I_{H}$ and $I_{V}$ in terms of $\Delta_{H}-\Gamma_{H}$ and $\Delta_{V}-\Gamma_{V}$. One finds

$$
\begin{gathered}
I_{H}=L_{V}\left\{\frac{\Delta_{H}-\Gamma_{H}}{D}\right\}-K_{V}\left\{\frac{\Delta_{V}-\Gamma_{V}}{D}\right\} \\
I_{V}=-\left(L_{H}+L_{C}+L_{F}\right)\left\{\frac{\Delta_{H}-\Gamma_{H}}{D}\right\}+\left(K_{H}+K_{C}+K_{F}\right)\left\{\frac{\Delta_{V}-\Gamma_{V}}{D}\right\}
\end{gathered}
$$

where

$$
D=\left(K_{H}+K_{C}+K_{F}\right) L_{V}-\left(L_{H}+L_{C}+L_{F}\right) K_{V} .
$$

Finally, using in (3) the values of $I_{0}, I_{R}$ and $I_{H}$ obtained from (11) and (12), one obtains currents $I_{C}$ and $I_{F}$. Thus, given $\xi_{H}, \xi_{V}, R_{0}$ and $R$, currents $I_{H}, I_{V}, I_{C}$ and $I_{F}$ are calculated according to equations (6-14) and $(3)$. 


\section{Chromaticities and Currents in the Original Chrom Control Program}

The chromaticities and currents are programmed in the Booster Chrom Control part of the Optics Control application. The original Chrom Control program dealt with just two sextupole strings; the vertical string and the original horizontal string consisting of all 24 horizontal sextupoles. The chromaticities in this case are given by equations (1) and (2) with

$$
I_{C}=I_{F}=I_{H} .
$$

One then has

$$
\begin{aligned}
\xi_{H} & =\xi_{H}^{0}+\frac{S}{B \rho}\left\{\left(K_{H}+K_{C}+K_{F}\right) I_{H}+K_{V} I_{V}\right\} \\
\xi_{V} & =\xi_{V}^{0}+\frac{S}{B \rho}\left\{\left(L_{H}+L_{C}+L_{F}\right) I_{H}+L_{V} I_{V}\right\} .
\end{aligned}
$$

These equations and their inverse give the chromaticities and currents as calculated by the original program.

\section{Formulae for the New Chrom Control Program}

The new Chrom Control program is a version of the original one with modifications to include the new sextupole strings and the resonance parameters. Both the new and original programs deal with the normalized chromaticities

$$
C_{H}=\frac{1}{Q_{H}} \xi_{H}, \quad C_{H}^{0}=\frac{1}{Q_{H}} \xi_{H}^{0}, \quad C_{V}=\frac{1}{Q_{V}} \xi_{V}, \quad C_{V}^{0}=\frac{1}{Q_{V}} \xi_{V}^{0}
$$

where $Q_{H}$ and $Q_{V}$ are the horizontal and vertical tunes. Both programs use parameters

$$
\begin{array}{ll}
A_{11}=\frac{1}{Q_{H}}\left(\frac{S}{B_{0} \rho}\right)\left(K_{H}+K_{C}+K_{F}\right), & A_{12}=\frac{1}{Q_{H}}\left(\frac{S}{B_{0} \rho}\right) K_{V} \\
A_{21}=\frac{1}{Q_{V}}\left(\frac{S}{B_{0} \rho}\right)\left(L_{H}+L_{C}+L_{F}\right), & A_{22}=\frac{1}{Q_{V}}\left(\frac{S}{B_{0} \rho}\right) L_{V}
\end{array}
$$

which are specified by the user as functions of the magnetic field $B=B \rho / \rho$. Here $\rho=13.8656 \mathrm{~m}$ is the nominal radius of curvature in the 
Booster dipoles. The field $B_{0}$ is a reference field chosen to be $0.1563 \mathrm{~T}$.

The values of $A_{11}, A_{12}, A_{21}$ and $A_{22}$ are given in Appendix III.

The new program requires additional parameters

$$
\begin{array}{ll}
R_{11}=\frac{1}{Q_{H}}\left(\frac{S}{B_{0} \rho}\right)\left(K_{C}+K_{F}\right), & R_{12}=\frac{1}{Q_{H}}\left(\frac{S}{B_{0} \rho}\right)\left(K_{C}-K_{F}\right) \\
R_{21}=\frac{1}{Q_{V}}\left(\frac{S}{B_{0} \rho}\right)\left(L_{C}+L_{F}\right), & R_{22}=\frac{1}{Q_{V}}\left(\frac{S}{B_{0} \rho}\right)\left(L_{C}-L_{F}\right)
\end{array}
$$

and

$$
A_{0}=\left(\frac{S}{B_{0} \rho}\right) K_{0}, \quad A_{R}=\left(\frac{S}{B_{0} \rho}\right) K_{R}
$$

These are also specified by the user as functions of the field $B$. The values of $R_{11}, R_{12}, R_{21}$ and $R_{22}$ are given in Appendix III. The values of $A_{0}$ and $A_{R}$ are to be chosen to give $R_{0}$ and $R$ convenient units.

Note that in equations (18-22), $Q_{H}$ and $Q_{V}$ are neither the measured tunes nor are they the tunes specified in the Booster Tune Control part of the Optics Control application. Instead, they are fixed at the values $Q_{H}=4.82$ and $Q_{V}=4.83$. Thus, to recover the values of $\xi_{H}$ and $\xi_{V}$, one has to multiply $C_{H}$ and $C_{V}$ by these fixed values. (One could avoid having to do this by simply dealing with $\xi_{H}$ and $\xi_{V}$ instead of the normalized chromaticities $C_{H}$ and $C_{V}$.)

In terms of the user specified parameters, equations (1), (2) and (5) become

$$
\begin{gathered}
C_{H}=C_{H}^{0}+\frac{B_{0}}{B}\left\{A_{11} I_{H}+A_{12} I_{V}+R_{11} I_{0}+R_{12} I_{R}\right\} \\
C_{V}=C_{V}^{0}+\frac{B_{0}}{B}\left\{A_{21} I_{H}+A_{22} I_{V}+R_{21} I_{0}+R_{22} I_{R}\right\} \\
R_{0}=\frac{B_{0}}{B} A_{0} I_{0}, \quad R=\frac{B_{0}}{B} A_{R} I_{R}
\end{gathered}
$$

where

$$
I_{0}=-I_{H}+\left(I_{C}+I_{F}\right) / 2, \quad I_{R}=\left(I_{C}-I_{F}\right) / 2 .
$$

These equations give the normalized chromaticities and resonance parameters in terms of currents $I_{H}, I_{V}, I_{C}$ and $I_{F}$. Their inverse gives the currents in terms of the chromaticities and resonance parameters. The 
formulae for going back and forth between chromaticities, resonance parameters, and currents are summarized in the following two subsections. The user interface for programming $C_{H}, C_{V}, R_{0}, R, I_{H}, I_{V}, I_{C}$ and $I_{F}$ is shown schematically in Figure 5.

\subsection{Formulae for Going from Currents to Chromaticities and Resonance Parameters}

Here one computes

$$
I_{0}=-I_{H}+\left(I_{C}+I_{F}\right) / 2, \quad I_{R}=\left(I_{C}-I_{F}\right) / 2
$$

and then

$$
\begin{gathered}
C_{H}=C_{H}^{0}+\frac{B_{0}}{B}\left\{A_{11} I_{H}+A_{12} I_{V}+R_{11} I_{0}+R_{12} I_{R}\right\} \\
C_{V}=C_{V}^{0}+\frac{B_{0}}{B}\left\{A_{21} I_{H}+A_{22} I_{V}+R_{21} I_{0}+R_{22} I_{R}\right\} \\
R_{0}=\frac{B_{0}}{B} A_{0} I_{0}, \quad R=\frac{B_{0}}{B} A_{R} I_{R} .
\end{gathered}
$$

\subsection{Formulae for Going from Chromaticities and Resonance Parameters to Currents}

Here one computes

$$
\begin{gathered}
I_{0}=\frac{1}{A_{0}} \frac{B}{B_{0}} R_{0}, \quad I_{R}=\frac{1}{A_{R}} \frac{B}{B_{0}} R \\
D_{H}=C_{H}-C_{H}^{0}-\frac{B_{0}}{B}\left\{R_{11} I_{0}+R_{12} I_{R}\right\} \\
D_{V}=C_{V}-C_{V}^{0}-\frac{B_{0}}{B}\left\{R_{21} I_{0}+R_{22} I_{R}\right\} .
\end{gathered}
$$

Equations (24) and (25) then give

$$
\frac{B_{0}}{B}\left\{A_{11} I_{H}+A_{12} I_{V}\right\}=D_{H}
$$




$$
\frac{B_{0}}{B}\left\{A_{21} I_{H}+A_{22} I_{V}\right\}=D_{V} .
$$

These may be inverted to give

$$
\begin{gathered}
I_{H}=\frac{1}{D}\left\{A_{22} D_{H}-A_{12} D_{V}\right\} \frac{B}{B_{0}} \\
I_{V}=\frac{1}{D}\left\{-A_{21} D_{H}+A_{11} D_{V}\right\} \frac{B}{B_{0}}
\end{gathered}
$$

where

$$
D=A_{11} A_{22}-A_{12} A_{21}
$$

Finally, one computes

$$
I_{C}=I_{H}+I_{0}+I_{R}, \quad I_{F}=I_{H}+I_{0}-I_{R} .
$$

Note that when $R=R_{0}=0$, one has $I_{R}=I_{0}=0$ and $I_{C}=I_{F}=I_{H}$. The currents in the $\mathrm{C}$ and $\mathrm{F}$ strings must then track the current in the horizontal string. This is the normal mode of operation when the $3 Q_{H}=13$ resonance is not being excited for resonant extraction. When $R$ is nonzero (as it must be during resonant extraction), one would generally like to keep $R_{0}=0$, but this may require that either $I_{C}$ or $I_{F}$ be larger than the available current. In such situations, $R_{0}$ must be adjusted to some nonzero value to keep the currents within the bounds of what is available. Figure 6 shows how the currents might be programmed for a typical NSRL magnet cycle.

\section{Appendix I}

The horizontal and vertical chromaticities in Booster are given by $[2,3]$

$$
\xi_{H}=\xi_{H}^{0}+\frac{1}{4 \pi} \frac{S}{B \rho} \sum_{j} \beta_{H}^{j} D_{j} I_{j}
$$

and

$$
\xi_{V}=\xi_{V}^{0}-\frac{1}{4 \pi} \frac{S}{B \rho} \sum_{j} \beta_{V}^{j} D_{j} I_{j}
$$

where the sums are taken over all 48 sextupoles. Here $\xi_{H}^{0}$ and $\xi_{V}^{0}$ are the bare chromaticities and $B \rho$ is the magnetic rigidity. $\beta_{H}^{j}, \beta_{V}^{j}$, and $D_{j}$ are 
respectively the horizontal and vertical beta functions and the periodic dispersion at the $j$ th sextupole. $I_{j}$ is the current in the $j$ th sextupole. The integrated strength per unit current of each sextupole is [4]

$$
S=\left|B_{2} L\right|=2 \times 6.566 \times 10^{-3}(\mathrm{~T} / \mathrm{m}) / \mathrm{A}
$$

where $B=B_{2} x^{2} / 2$ is the magnetic field (per unit current) on the midplane of the sextupole, $x$ is the horizontal coordinate on the midplane, and $L$ is the magnetic length.

Let us now define

$$
\begin{gathered}
K_{H}=\frac{1}{4 \pi} \sum_{h} \beta_{H}^{h} D_{h}, \quad K_{V}=-\frac{1}{4 \pi} \sum_{v} \beta_{H}^{v} D_{v}, \\
K_{C}=\frac{1}{4 \pi} \sum_{c} \beta_{H}^{c} D_{c}, \quad K_{F}=\frac{1}{4 \pi} \sum_{f} \beta_{H}^{f} D_{f}, \\
L_{H}=-\frac{1}{4 \pi} \sum_{h} \beta_{V}^{h} D_{h}, \quad L_{V}=\frac{1}{4 \pi} \sum_{v} \beta_{V}^{v} D_{v},
\end{gathered}
$$

and

$$
L_{C}=-\frac{1}{4 \pi} \sum_{c} \beta_{V}^{c} D_{c}, \quad L_{F}=-\frac{1}{4 \pi} \sum_{f} \beta_{V}^{f} D_{f}
$$

where the sum over $h$ is the sum over all horizontal sextupoles except SHC8, SHE4, SHF8, and SHB4; the sum over $v$ is the sum over all vertical sextupoles; the sum over $c$ is the sum over sextupoles SHC8 and SHE4; and the sum over $f$ is the sum over sextupoles SHF8 and SHB4. Equations (41) and (42) then become

$$
\xi_{H}=\xi_{H}^{0}+\frac{S}{B \rho}\left(K_{H} I_{H}+K_{V} I_{V}+K_{C} I_{C}+K_{F} I_{F}\right)
$$

and

$$
\xi_{V}=\xi_{V}^{0}+\frac{S}{B \rho}\left(L_{H} I_{H}+L_{V} I_{V}+L_{C} I_{C}+L_{F} I_{F}\right)
$$

where the four currents $I_{H}, I_{V}, I_{C}$, and $I_{F}$ are defined in Sections 1 and 3. Note that the signs of $K_{H}, K_{C}, K_{F}, L_{H}, L_{C}, L_{F}$ are such that positive currents $I_{H}, I_{C}, I_{F}$ increase the horizontal chromaticity and decrease the vertical chromaticity. The signs of $K_{V}$ and $L_{V}$ are such that positive current $I_{V}$ increases the vertical chromaticity and decreases the horizontal chromaticity. 
The sums in (44-47) are readily evaluated using the MAD code. One finds

$$
\begin{array}{ll}
K_{H}=\frac{472.86}{4 \pi}, & K_{V}=-\frac{122.90}{4 \pi}, \quad K_{C}=K_{F}=\frac{47.267}{4 \pi} \\
L_{H}=-\frac{162.03}{4 \pi}, & L_{V}=\frac{355.38}{4 \pi}, \quad L_{C}=L_{F}=-\frac{16.067}{4 \pi}
\end{array}
$$

and

$$
K_{H}+K_{C}+K_{F}=\frac{567.39}{4 \pi}, \quad L_{H}+L_{C}+L_{F}=-\frac{194.16}{4 \pi}
$$

where the units are meters squared. Here the horizontal and vertical tunes were taken to be $Q_{H}=4.82$ and $Q_{V}=4.83$. Note that it follows from superperiod symmetry that

$$
K_{C}=K_{F}, \quad L_{C}=L_{F} .
$$

Since the constants defined in (44-47) depend on the beta functions and dispersion, they will depend on the machine tunes. This dependence could be put into the Chrom Control program by means of a lookup table which gives the values of the constants for various tunes.

\section{$7 \quad$ Appendix II}

As defined by (1) and (2), the bare chromaticities, $\xi_{H}^{0}$ and $\xi_{V}^{0}$, are what one would measure with zero current in the four sextupole strings. There are several contributions to $\xi_{H}^{0}$ and $\xi_{V}^{0}$ which may be expressed by writing

$$
\xi_{H}^{0}=\xi_{H}^{N}+\frac{1}{B \rho}\left(A_{H}+\dot{B} B_{H}\right)+E_{H}
$$

and

$$
\xi_{V}^{0}=\xi_{V}^{N}+\frac{1}{B \rho}\left(A_{V}+\dot{B} B_{V}\right)+E_{V}
$$

Here $\xi_{H}^{N}$ and $\xi_{V}^{N}$ are the so called natural chromaticities $[2,3]$ due to the intrinsic quadrupole structure of the lattice. (They do not depend on sextupole fields.) For $Q_{H}=4.82$ and $Q_{V}=4.83$, the MAD code gives

$$
\xi_{H}^{N}=-4.941, \quad \xi_{V}^{N}=-5.258 .
$$

The $A_{H}$ and $A_{V}$ terms are due to remanent sextupole fields. The $B_{H}$ and $B_{V}$ terms are due to sextupole fields that are proportional to $\dot{B}=d B / d t$. 
The values of $A_{H}, A_{V}, B_{H}$, and $B_{V}$ must be determined from measurements. The $E_{H}$ and $E_{V}$ terms are due to sextupole fields in the Booster dipoles that scale with the magnet excitation. For given tunes, $E_{H}$ and $E_{V}$ are assumed to be constant until the dipoles begin to saturate. For $Q_{H}=4.82$ and $Q_{V}=4.83$, the MAD code gives (with no dipole saturation)

$$
E_{H}=-2.342, \quad E_{V}=+2.194
$$

Thus we have (with no dipole saturation)

$$
\xi_{H}^{0}=-7.283+\frac{1}{B \rho}\left(A_{H}+\dot{B} B_{H}\right)
$$

and

$$
\xi_{V}^{0}=-3.064+\frac{1}{B \rho}\left(A_{V}+\dot{B} B_{V}\right) .
$$

In the present Chrom Control program, the normalized bare chromaticities

$$
C_{H}^{0}=\frac{1}{Q_{H}} \xi_{H}^{0}, \quad C_{V}^{0}=\frac{1}{Q_{V}} \xi_{V}^{0}
$$

are specified by the user as functions of the magnetic field $B$.

\section{Appendix III}

Using (50-52) in (19-22), one finds

$$
\begin{gathered}
A_{11}=0.0568, \quad A_{12}=-0.0123, \quad A_{21}=-0.0194, \quad A_{22}=0.0355 \\
R_{11}=0.00946, \quad R_{12}=0.0, \quad R_{21}=-0.00321, \quad R_{22}=0.0 .
\end{gathered}
$$

Here the units are $\mathrm{A}^{-1}$ and we have used $Q_{H}=4.82, Q_{V}=4.83$, $B_{0}=0.1563 \mathrm{~T}, \rho=13.8656 \mathrm{~m}$, and $S=2 \times 6.566 \times 10^{-3}(\mathrm{~T} / \mathrm{m}) / \mathrm{A}$. In the present Chrom Control program, the archived values of $A_{11}, A_{12}, A_{21}, A_{22}$ are

$$
A_{11}=0.059, \quad A_{12}=-0.013, \quad A_{21}=-0.019, \quad A_{22}=0.036 .
$$

These are in good agreement with the calculated values.

Note that even though $A_{11}, A_{12}, A_{21}, A_{22}, R_{11}, R_{12}, R_{21}, R_{22}, A_{0}$ and $A_{R}$ can be programmed as arbitrary functions of $B$ in the Chrom Control program, they are simply programmed to be constant functions of $B$. 


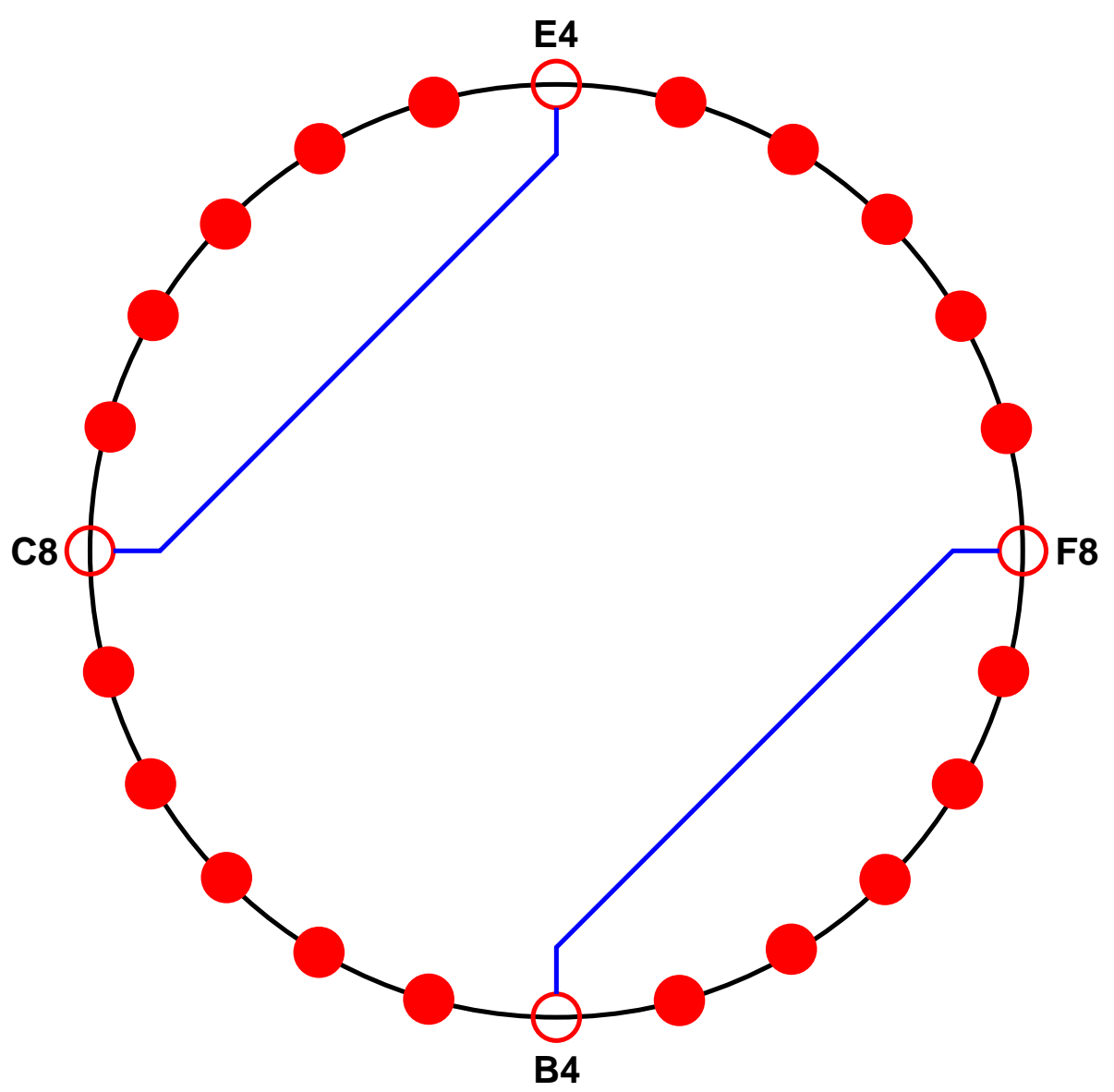

Figure 1: Horizontal Sextupoles in the Booster Ring. The open circles show the positions of the four sextupoles used for resonant extraction. The filled circles show the positions of the remaining 20 horizontal sextupoles. 


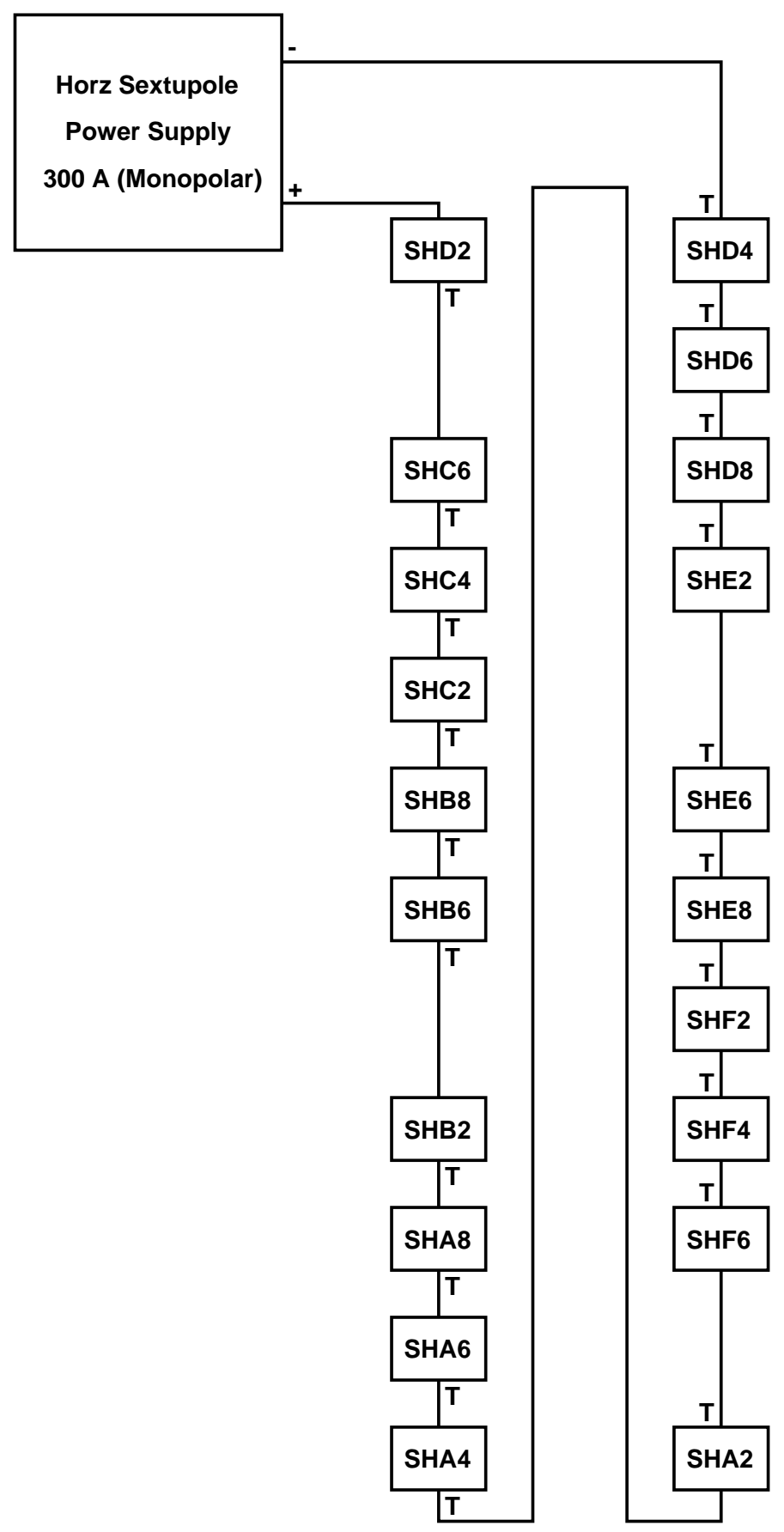

Figure 2: Wiring Diagram for the Horizontal Sextupole String. Here, in accordance with the definitions of Ref. [1], the monopolar power supply puts the sextupoles in "B" polarity. This increases the horizontal chromaticity. 


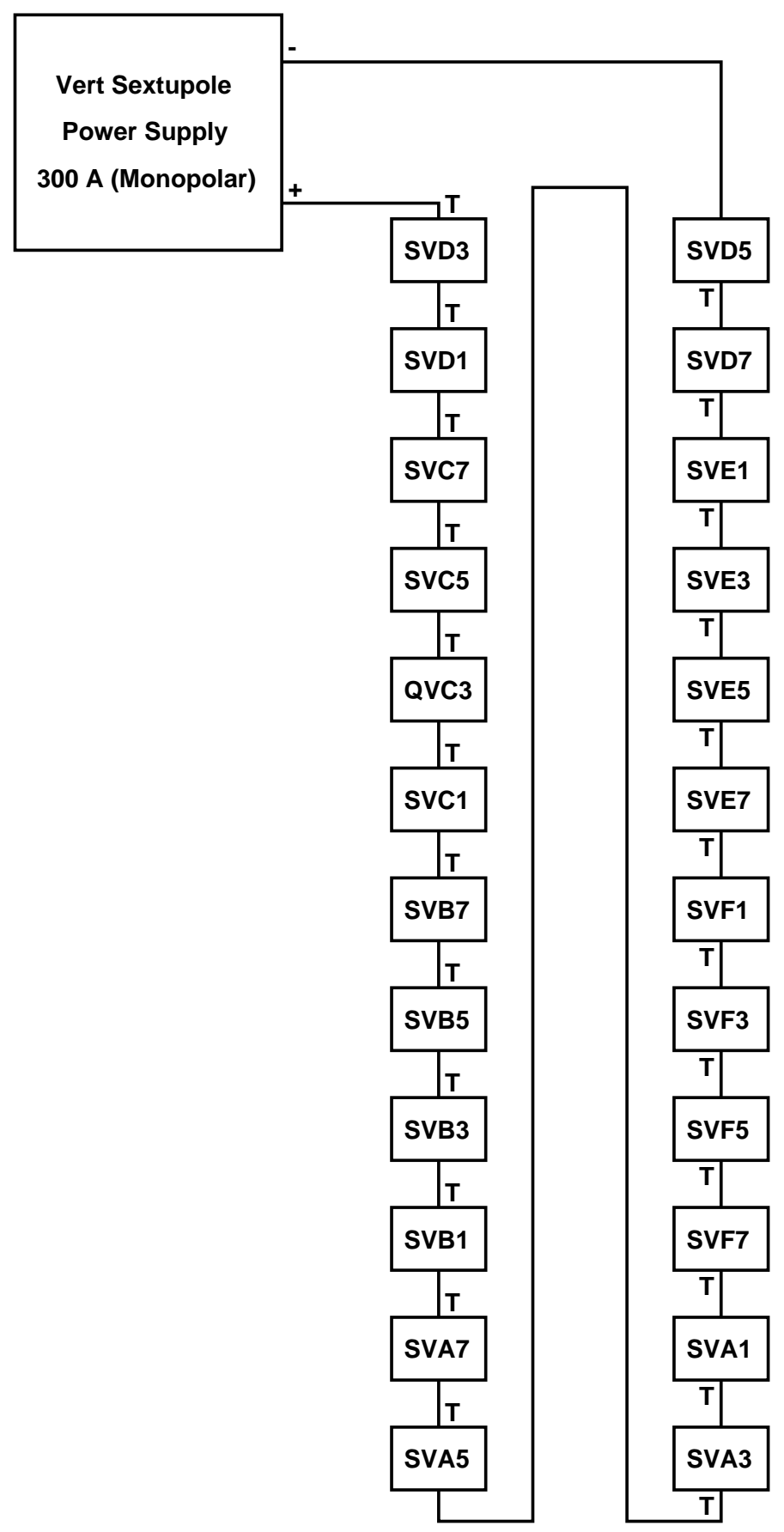

Figure 3: Wiring Diagram for the Vertical Sextupole String. Here, in accordance with the definitions of Ref. [1], the monopolar power supply puts the sextupoles in "A" polarity. This increases the vertical chromaticity. 

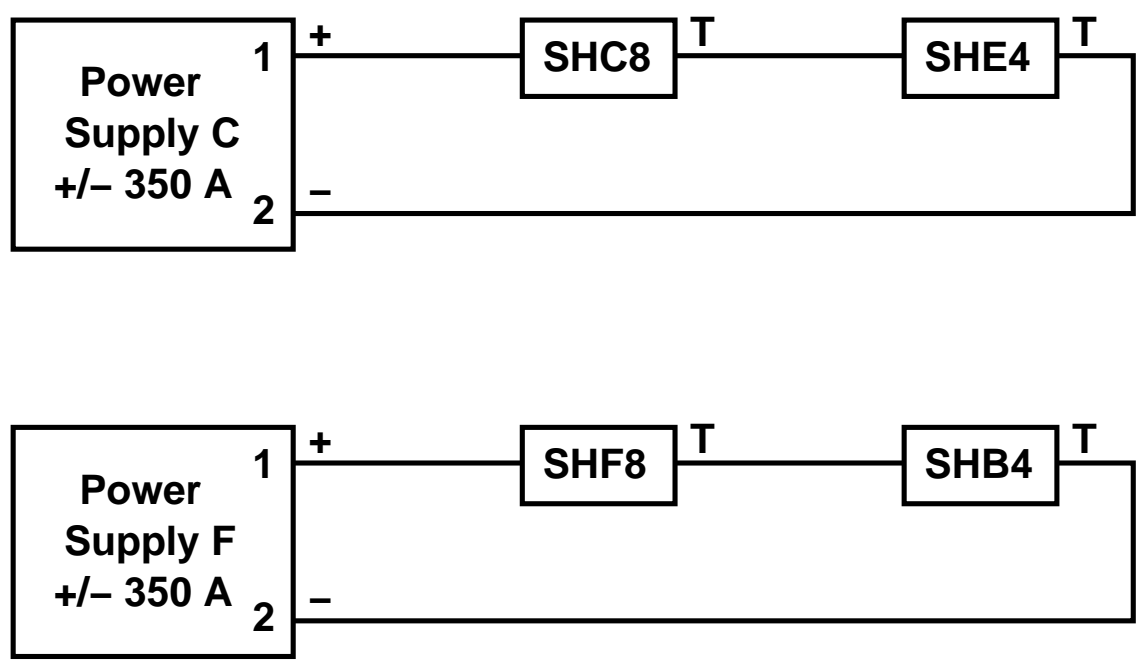

Figure 4: Wiring Diagram for the $\mathrm{C}$ and F Strings. In accordance with the conventions of Ref. [1], a positive reference at the power supply input produces a positive current out of the terminal labeled " 1 ". This puts the sextupoles in the strings in "B" polarity. The $Q_{H}=4+1 / 3$ resonance is excited by powering the two strings with opposite polarity. Note that in the control system, power supplies $\mathrm{C}$ and F are called "b-sxr1-ps" and "b-sxr2-ps" respectively. 
IH, IV, IC, and IF versus Time

ChromH, ChromV, R, and R0 versus Time

\begin{tabular}{|l|}
\hline Time \\
\hline
\end{tabular}

Figure 5: New User Interface 




Figure 6: Actual user interface showing how currents $I_{H}, I_{V}, I_{C}, I_{F}$ and parameters $C_{H}, C_{V}, R, R_{0}$ might be programmed for a typical NSRL magnetic cycle. 


\section{References}

[1] E. Bleser, "Booster Polarity Standards", Booster Technical Note 180, October 30, 1990

[2] D.A. Edwards and M.J. Syphers, "An Introduction to the Physics of High Energy Accelerators", John Wiley \& Sons, Inc., New York, 1993, p. 98

[3] A.W. Chao and M. Tigner (editors), "Handbook of Accelerator Physics and Engineering", World Scientific, Singapore, 1999, p. 50

[4] E. Bleser, "Booster Sextupole Production Measurements", Booster Technical Note 182, March 13, 1991 